\title{
A Bio-inspired Robust 3D Plume Tracking Strategy Using Mobile Sensor Networks
}

\author{
Wencen Wu, Dongsik Chang, and Fumin Zhang
}

\begin{abstract}
We develop a robust plume tracking strategy using mobile sensor networks in three dimensional (3D) fields. Inspired by the plume tracking behavior of blue crabs, we propose a stochastic model of plume spikes detected by sensing agents based on the Poisson counting process, which enables us to transform the turbulent plume field detected by sensing agents to a continuously-differential field, the minimum of which is considered as a source in the field, and shares the same location with the plume source. The transformation allows us to design and analyze source-seeking algorithms in smooth fields instead of in turbulent fields with higher fluctuation spatially and temporally. Inspired by fish schools that seek darker (shaded) regions in environments with complex lighting variations, we develop a distributed source-seeking algorithm using mobile sensor networks without explicit gradient estimation. The velocity of each agent is designed using only the measurements taken by the agent and the relative positions to its neighboring agents. We prove that, using this design, the moving direction of a three-agent group will converge to the opposite gradient direction of the field, thus, the group moves towards a source in the field. We also prove that the tracking system is input-to-state stable (ISS), indicating that the system is robust to disturbances. We then generalize the design to $\mathrm{N}$ agent groups, and demonstrate the strategy in both smooth fields and turbulent fields in simulations.
\end{abstract}

\section{INTRODUCTION}

Exploration tasks that require mobile sensing agents to localize and identify a feature of interest (source) in scalar fields such as chemical and temperature fields are of great concern to researchers and are important in many scenarios. For example, locating the position of oil spills and identifying the origin of a wild fire. To detect and track a source in a scalar field, various algorithms have been proposed in the literature. For example, references [1]-[5] introduced collective gradient estimating and tracking algorithms using multiple sensing agents, and references [6]-[10] present source-seeking algorithms using a single robot. Most of the work focus on two-dimensional (2D) fields, few of them extends the results to three-dimensional (3D) fields [11]. Reference [5] introduces a source-seeking algorithm using a group of mobile sensing agents in a distributed fashion with no explicit gradient estimation in a 2D field. The algorithm is inspired by fish schools that are able to perform gradient

The research work is supported by ONR grants N00014-09-1-1074 and N00014-10-10712 (YIP), and NSF grants ECCS- 0841195 (CAREER), CNS-0931576, OCE-1032285, and IIS-1319874. Wencen Wu, Dongsik Chang, and Fumin Zhang are with the School of Electrical and Computer Engineering, Georgia Institute of Technology, Atlanta, GA, 30332, USA wwencen3, dsfrancis3, fumindgatech.edu. The authors would like to thank Drs. D. Webster, M. Weissburg, and M. Malisoff for their help on improving the paper. tracking to locate darker (shaded) regions in complex light environments even if the field is time-varying [12].

To locate sources in fluid flow environments, one may not expect a well-behaved scalar field. When the Reynolds number are low, gradients may be well-defined. However, At medium to high Reynolds numbers, chemical or particle dispersion is dominated by turbulent mixing, which produces scalar fields that fluctuate rapidly in spatial and temporal scales [13]-[15]. The source seeking problem in such fields is more challenging due to the lack of analytical methods that describe the "plume-like" distribution. Gradient-based methods developed for smooth scalar field can not be directly applied. Various algorithms are developed and inspired by the extraordinary capability of plume tracking by moths, blue crabs, and bees [16]-[22]. Most of them apply to single agent.

A stochastic model has been developed in [23] such that plume spikes detected by sensing agents can be estimated based on the Poisson counting process, which enables the transformation of a turbulent plume field to a continuouslydifferentiable field, the minimum of which is considered as a source in the field, and shares the same location with the plume source. The field transformation is inspired by behaviors of blue crabs and the sensor-mediated plume tracking algorithm in a controlled turbulent flow environment developed by Webster et al. [19]. Then, the source seeking strategy developed in [5] has been utilized by mobile sensing agents, which are able to locate the plume source in a turbulent field.

In this paper, we extend the distributed source-seeking strategy in [5] to 3D, and demonstrate that the source-seeking in smooth fields and the plume tracking in turbulent fields can be unified in 3D by performing a field transformation similar to [23]. After a turbulent field is transformed to a smooth field, we design source-seeking strategy to control the velocities of the agents and analysis the convergence of the strategy in a smooth field. We decompose the velocity of each agent into three parts along each direction of a right-handed coordinate frame determined by a three-agent group. The three-agent group is controlled to remain a constant formation so that they are considered as a rigid body. Under the assumption that the agents do not share measurements with other agents, the forward speed of each agent is designed to be proportional to the field values. In other words, the agents speed up when the field values increase, and slow down when the field values decrease. With the help of differential geometry, we prove that the moving direction of the center of the group will converge to 
the opposite direction of the gradient, thus, moving towards a local minimum (source) of the field, and the system is input-to-state stable (ISS). The convergence of the formation control laws is proved using shape variables as described in [24]-[26].

The contributions of this work are (1) by extending the results in [23] from $2 \mathrm{D}$ to $3 \mathrm{D}$, we transform a turbulent field into a continuously-differentiable field, which is more amenable to analysis; (2) we introduce a distributed plume tracking strategy in 3D turbulent fields using mobile sensor networks that requires no communication among agents; and (2) by proving the input-to-state stability of the tracking system, we are able to show that the plume tracking strategy is robust to bounded disturbances.

The rest of the paper is organized as follows. Section II introduces the problem formulation and the field transformation. Section III presents source-seeking control law design for three-agent groups in $3 \mathrm{D}$, the convergence proof, the generalization to $N$-agent groups, and the simulation results in smooth fields. Section IV introduces plume tracking strategy in turbulent fields and illustrates the simulation results. Section $\mathrm{V}$ provides concluding remarks and future work.

\section{Problem Formulation}

Let $z(\mathbf{r}), \mathbf{r} \in R^{3}$ represent a scaler field, in which every location of the field associates with a scalar value such as temperature, light intensity, or chemical concentration. Consider a group of $N$ sensing agents moving in the field and taking measurements of the field along their trajectories. The measurements are denoted by $y\left(\mathbf{r}_{i}\right)=z\left(\mathbf{r}_{i}\right)+v\left(\mathbf{r}_{i}\right), i=$ $1, \cdots, N$, in which $v\left(\mathbf{r}_{i}\right)$ is the noise term that may come from measuring process or the field. Let $\mathbf{r}_{i}$ represent the position and $\mathbf{v}_{i}$ the velocity of the $i$ th agent. Suppose the motion of each agent in the group satisfies $\dot{\mathbf{r}}_{i}=\mathbf{v}_{i}, i=1, \cdots, N$. Denote the position and velocity of the group center as $\mathbf{r}_{c}$ and $\mathbf{v}_{c}$, respectively. Then, we derive $\mathbf{r}_{c}=\frac{1}{N} \sum_{i=1}^{N} \mathbf{r}_{i}$, and $\mathbf{v}_{c}=\frac{1}{N} \sum_{i=1}^{N} \mathbf{v}_{i}$.

We assume the agents can sense their relative positions to neighboring agents using sensors such as sonar, radar, and camera, but they do not share measurements with other agents. If $z(\mathbf{r})$ is generated by a turbulent field with a chemical plume source, as illustrated in Fig. 1, the goal is to control the group of mobile sensing agents to detect and track the plume source from initial locations that are downstream to the plume source.

In [23], an approach that transforms a turbulent flow field detected by agents into a smooth field sharing the same source has been proposed. Suppose the transformed smooth field is at least class $C^{1}$ with minimum $z_{\min }$ and maximum $z_{\max }$, in which $z_{\min } \geq 0$, then the plume tracking in a turbulent field is converted to the source-seeking problem in a transformed smooth field. The local minimum $z_{\min } \geq$ 0 is considered as a source of the field. In this section, we introduce the transformation of the field in 3D, which motivates our development of source-seeking strategy in smooth fields.

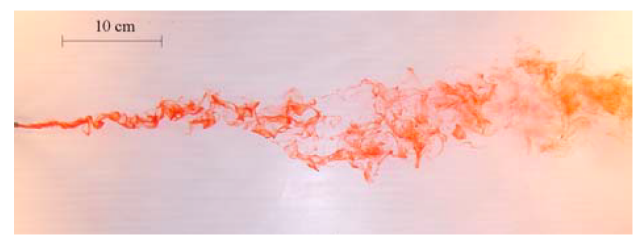

Fig. 1. A turbulent plume field in 2D. Adapted from [19].

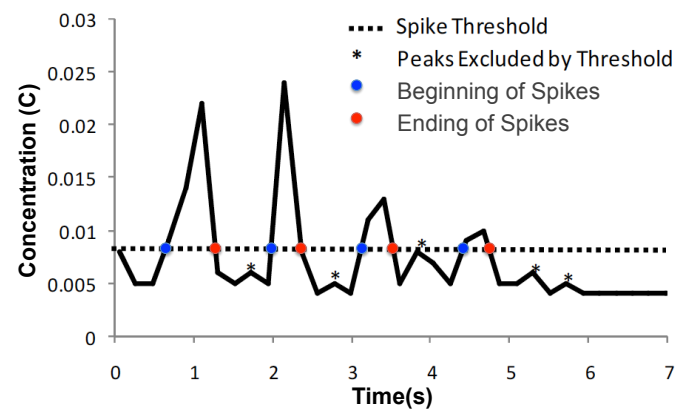

Fig. 2. Concentration measured along the trajectory of a moving sensor in a turbulent chemical plume. Adapted from [19].

\section{A. Modeling Plume Spikes}

Based on the experimental data in [19], [27], we notice that when a mobile sensor moves in a plume at constant speed, the measurement of concentration along its trajectory will display spike-like structure as shown in Fig. 2. A spike can then be detected by comparing the measurement with a given threshold. The occurrences of spikes can be modeled as a Poisson process. Another important observation is that as the sensor gets closer to the plume source, the average frequency of the spike occurrence becomes lower, but the duration of each spike becomes longer.

Based on the knowledge of spikes in [19], we introduce a stochastic model that describes the random occurrences of spikes along the trajectory of an agent. We define the spike indicator $s=\left\{s_{1}, s_{2}\right\}$, where $s_{1}, s_{2} \in \mathbb{Z}$, that identifies the status of spike detected by a sensing agent: $s=s_{1}$ indicates no spike at the current position of the agent, and $s=s_{2}$ indicates otherwise. The spike indicator $s$ satisfies the following stochastic differential equation driven by Poisson counters:

$$
d s=\left(s_{1}-s\right) d N_{12}+\left(s_{2}-s\right) d N_{21}
$$

where $d N_{12}, d N_{21}$ are Poisson jump processes that trigger the state $s$ to jump from $s_{1}$ to $s_{2}$ and from $s_{2}$ back to $s_{1}$, respectively. Along the trajectory, a state jump from $s_{1}$ to $s_{2}$ indicates the beginning of a spike and a jump from $s_{2}$ to $s_{1}$ indicates the ending of a spike.

The state transitions of the spike indicator happen randomly, and the rates $\lambda_{12}$ and $\lambda_{21}$, which are associated with the jump processes $d N_{12}$ and $d N_{21}$, determine how frequently state transitions happen. In other words, the rates affect the duration of a spike as well as the expected frequency of the spike occurrences. Based on the observation from [19], $\lambda_{12}$ 
and $\lambda_{21}$ can be modeled as follows. For the $i$ th sensing agent in a field, let $\lambda_{12, i}$ and $\lambda_{21, i}$ be the rates of the Poisson jump process. Suppose the chemical source is located at position $\mathbf{r}_{0}$. Let the distance between the agent and the source be $\left\|\mathbf{r}_{i}-\mathbf{r}_{0}\right\|$. Then, we assume that $\lambda_{12, i}$ is a monotonically increasing function of the the distance and $\lambda_{21, i}$ is inverse proportional to the distance, e.g.,

$$
\begin{aligned}
& \lambda_{12, i}=k_{\lambda} \frac{1}{g\left(\left\|\mathbf{r}_{i}-\mathbf{r}_{0}\right\|\right)}, \\
& \lambda_{21, i}=k_{\lambda}^{\prime} g\left(\left\|\mathbf{r}_{i}-\mathbf{r}_{0}\right\|\right),
\end{aligned}
$$

in which $k_{\lambda}$ and $k_{\lambda}^{\prime}$ are positive constants chosen by design. $g\left(\left\|\mathbf{r}_{i}-\mathbf{r}_{0}\right\|\right) \neq 0$ is assumed to be a monotonically increasing function of $\left\|\mathbf{r}_{i}-\mathbf{r}_{0}\right\|$.

Equation (1) in association with the rates $\lambda_{12, i}, \lambda_{21, i}$ in Equation (2) for the jump processes model the timing of spikes in a turbulent field. Define a function

$$
f\left(\mathbf{r}_{i}\right)=\frac{\lambda_{12, i}+\lambda_{21, i}}{\lambda_{12, i}}=\frac{k_{\lambda}^{\prime}}{k_{\lambda}} g\left(\left\|\mathbf{r}_{i}-\mathbf{r}_{0}\right\|\right)^{2}+1,
$$

which is a smooth function of distance $\left\|\mathbf{r}_{i}-\mathbf{r}_{0}\right\|$. One simple example for $g\left(\left\|\mathbf{r}_{i}-\mathbf{r}_{0}\right\|\right)$ is $g\left(\left\|\mathbf{r}_{i}-\mathbf{r}_{0}\right\|\right)=\left\|\mathbf{r}_{i}-\mathbf{r}_{0}\right\|$. Then, since the source is located at $\mathbf{r}_{0}$, where the function $f\left(\mathbf{r}_{i}\right)$ has a unique minimum value 1 , the problem of finding the plume source in the turbulent field is now equivalent to finding the minimum point in the smooth field $f(\mathbf{r})$.

\section{Source-SeEking In SMooth FIELdS}

In this section, we first introduce the control law design for a three-agent group seeking for a source in a smooth field, and prove the convergence of the moving direction of the group with the opposite direction of the gradient of the field. We then generalize the design to $\mathrm{N}$-agent groups and present simulation results.

\section{A. Control Law Design for Three-agent Groups}

Suppose the positions of the three agents $\mathbf{r}_{i}, i=1,2,3$, are not in a line, which indicates that the three agents determines a plane $M$. Define a right-handed coordinate frame $\left(\mathbf{q}, \mathbf{q}^{\perp}, \mathbf{n}\right)$ with the origin locating at $\mathbf{r}_{c}$ and $\mathbf{q}$ and $\mathbf{q}^{\perp}$ lying on the plane $M$. $\mathbf{n}$ is determined by $\mathbf{q} \times \mathbf{q}^{\perp}$, which is normal to the plane.

For each agent, we decompose the velocity into three parts along the directions of $\mathbf{q}, \mathbf{q}^{\perp}$, and $\mathbf{n}$, and denote them as $\mathbf{v}_{i, \mathbf{q}}$, $\mathbf{v}_{i, \mathbf{q}^{\perp}}$, and $\mathbf{v}_{i, \mathbf{n}}, i=1,2,3$, respectively. Fig. 3 illustrates the geometry of the three-agent group in $3 \mathrm{D}$ space. In the figure, $(\mathbf{X}, \mathbf{Y}, \mathbf{Z})$ is the inertial frame, and $\mathbf{N}=\frac{\nabla f\left(\mathbf{r}_{c}\right)}{\| \nabla f\left(\mathbf{r}_{c} \|\right.}$ indicates the gradient direction of the field $f(\mathbf{r})$ at location $\mathbf{r}_{c}$. Given the decomposition, we obtain the velocity of the $i$ th agent as

$$
\mathbf{v}_{i}=\mathbf{v}_{i, \mathbf{q}}+\mathbf{v}_{i, \mathbf{q}^{\perp}}+\mathbf{v}_{i, \mathbf{n}}=v_{i, \mathbf{q}} \mathbf{q}+v_{i, \mathbf{q}^{\perp}} \mathbf{q}^{\perp}+v_{i, \mathbf{n}} \mathbf{n},
$$

where $v_{i, \mathbf{q}}, v_{i, \mathbf{q}^{\perp}}$, and $v_{i, \mathbf{n}}$ are speed along directions $\mathbf{q}, \mathbf{q}^{\perp}$, and $\mathbf{n}$, respectively. Note that this decomposition decouples the normal component of the velocity $\mathbf{v}_{i, \mathbf{n}}$ from the tangential components $\mathbf{v}_{i, \mathbf{q}}$ and $\mathbf{v}_{i, \mathbf{q}^{\perp}}$, which allows us to analyze the stability of the normal and tangential modes separately.

We aim to control the agents to maintain a constant formation in plane $M$ and move towards the minimum of

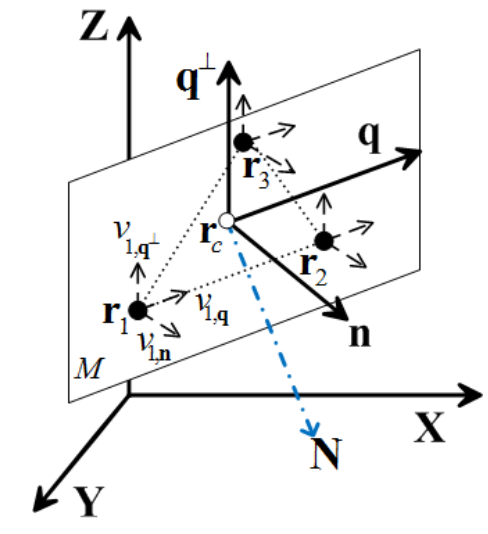

Fig. 3. The geometry of a three-agent group in inertial frame.

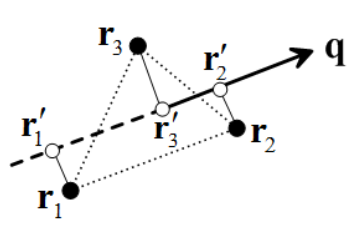

(a)

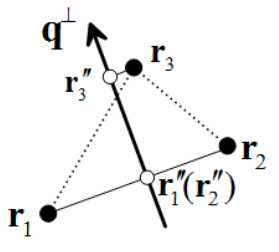

(b)
Fig. 4. The projections of $\mathbf{r}_{i}$ onto $\mathbf{q}$ (a) and $\mathbf{q}^{\perp}$ (b).

the field $f(\mathbf{r})$, which locates at $\mathbf{r}_{0}$. For now, we assume that the rates $\lambda_{12}$ and $\lambda_{21}$ are available to the agents along their trajectories so that the field $f(\mathbf{r})$ can be measured. We will discuss the estimation of the rates $\lambda_{12}$ and $\lambda_{21}$ in Section IV. Inspired by behaviors of fish schools [12], we design the speed in the normal direction $\mathbf{n}$ to be proportional to the field value $f\left(\mathbf{r}_{i}\right)$ as

$$
v_{i, \mathbf{n}}=k f\left(\mathbf{r}_{i}\right)+C
$$

in which $k>0$ and $C$ are constants.

For the tangential components of the velocity, let $\mathbf{r}_{i}^{\prime}$ be the projection of $\mathbf{r}_{i}$ onto vector $\mathbf{q}$, and $\mathbf{r}_{i}^{\prime \prime}$ be the projection of $\mathbf{r}_{i}$ onto vector $\mathbf{q}^{\perp}$, as illustrated in Fig. 4 (a) and (b), respectively. For agent $i$, we define sets $N_{i}$ and $N_{i}^{\perp}$ that contain the indices of the neighboring agents along directions $\mathbf{q}$ and $\mathbf{q}^{\perp}$. For example, for the three-agent group as shown in Fig. 4, $N_{1}=\{3\}, N_{2}=\{3\}, N_{3}=\{1,2\}, N_{1}^{\perp}=\{2,3\}$, $N_{2}^{\perp}=\{1,3\}, N_{3}^{\perp}=\{1,2\}$. The goal is to design $v_{i, \mathbf{q}}$ and $v_{i, \mathbf{q}^{\perp}}$ so that the relative distance from $\mathbf{r}_{i}^{\prime}$ to $\mathbf{r}_{j}^{\prime}, i \neq j$, converges to a constant $a_{i j}^{0}$, and the relative distance from $\mathbf{r}_{i}^{\prime \prime}$ to $\mathbf{r}_{j}^{\prime \prime}, i \neq j$, converges to a constant $b_{i j}^{0}$. Therefore, we design

$$
\begin{gathered}
v_{i, \mathbf{q}}=k_{1} \sum_{j \in N_{i}}\left(\left(\mathbf{r}_{j}-\mathbf{r}_{i}\right) \cdot \mathbf{q}-a_{j, i}^{0}\right), \\
v_{i, \mathbf{q}^{\perp}}=k_{2} \sum_{j \in N_{i}^{\perp}}\left(\left(\mathbf{r}_{j}-\mathbf{r}_{i}\right) \cdot \mathbf{q}^{\perp}-b_{j, i}^{0}\right),
\end{gathered}
$$

where $k_{1}>0$ and $k_{2}>0$ are constants, $a_{i, j}^{0}=-a_{j, i}^{0}$, and $b_{i, j}^{0}=$ $-b_{j, i}^{0}$. 


\section{B. Convergence Proof}

Given the controllers in Equations (5), (6), and (7), we first prove that the positions of the agents converge to a constant formation in plane $M$.

To prove the convergence under control (6), we define shape variables $s_{1}=\left(\mathbf{r}_{3}-\mathbf{r}_{1}\right) \cdot \mathbf{q}$ and $s_{2}=\left(\mathbf{r}_{2}-\mathbf{r}_{3}\right) \cdot \mathbf{q}$. We derive that

$$
\begin{aligned}
& \dot{s}_{1}=v_{3, \mathbf{q}}-v_{1, \mathbf{q}}=k_{1}\left(-2\left(s_{1}-a_{3,1}^{0}\right)+\left(s_{2}-a_{2,3}^{0}\right)\right), \\
& \dot{s}_{2}=v_{2, \mathbf{q}}-v_{3, \mathbf{q}}=k_{1}\left(\left(s_{1}-a_{3,1}^{0}\right)-2\left(s_{2}-a_{2,3}^{0}\right)\right) .
\end{aligned}
$$

Define $\mathbf{s}=\left(s_{1}, s_{2}\right)^{T}$ and $\mathbf{a}^{0}=\left(a_{3,1}^{0}, a_{2,3}^{0}\right)^{T}$, then, we obtain

$$
\dot{\mathbf{s}}=k_{1} A\left(\mathbf{s}-\mathbf{a}^{0}\right) \text {, }
$$

where $A=\left(\begin{array}{cc}-2 & 1 \\ 1 & -2\end{array}\right)$. Since the eigenvalues of $A$ are -1 and -3 , the state $\mathbf{s}$ of the system (9) will converge to the equilibrium $\mathbf{a}^{0}$ asymptotically. In other words, the relative distances among agents in direction $\mathbf{q}$ will converge to constants determined by $\mathbf{a}^{0}$. Define $\mathbf{b}^{0}=\left(b_{3,1}^{0}, b_{2,3}^{0}\right)^{T}$. Similar methods can be applied to prove the convergence of the relative distances among agents in direction $\mathbf{q}^{\perp}$ to $\mathbf{b}^{0}$ under control (7). Then, the agents will converge to a constant formation in plane $M$.

The constant formation can be treated as a rigid body, therefore, the frame $\left(\mathbf{q}, \mathbf{q}^{\perp}, \mathbf{n}\right)$ is considered as the body frame of the rigid body. We now prove that the moving direction of the rigid body converges to the opposite gradient direction at $\mathbf{r}_{c}$.

In the inertial frame, once the formation converges, $v_{i, \mathbf{q}}=$ $v_{i, \mathbf{q}^{\perp}}=0$. Then, the velocity of position $\mathbf{r}_{i}$ in the rigid body becomes $\mathbf{v}_{i}=v_{i, \mathbf{n}} \mathbf{n}$, and the velocity of the formation center is $\mathbf{v}_{c}=\frac{1}{3} \sum_{i=1}^{3} v_{i, \mathbf{n}} \mathbf{n}=v_{c, \mathbf{n}} \mathbf{n}$, which indicates that the moving direction of the rigid body coincides with the $\mathbf{n}$ axis of the body frame. In other words, the tangent vector of the trajectory of the rigid body aligns with $\mathbf{n}$. Define shape variables $\mathbf{N} \cdot \mathbf{n}, \mathbf{N} \cdot \mathbf{q}$, and $\mathbf{N} \cdot \mathbf{q}^{\perp}$, where $\mathbf{N}=\frac{\nabla f\left(\mathbf{r}_{c}\right)}{\left\|\nabla f\left(\mathbf{r}_{c}\right)\right\|}$ [24], [28]. Note that the shape variables satisfy the relationship

$$
\left(\mathbf{N} \cdot \mathbf{q}^{\perp}\right)^{2}=1-(\mathbf{N} \cdot \mathbf{q})^{2}-(\mathbf{N} \cdot \mathbf{n})^{2} .
$$

We aim to prove that as time $t \rightarrow \infty, \mathbf{N} \cdot \mathbf{n}$ converges to -1 . Since we have $\frac{d(\mathbf{N} \cdot \mathbf{n})}{d t}=\mathbf{N} \cdot \dot{\mathbf{n}}+\dot{\mathbf{N}} \cdot \mathbf{n}$, the first step is to find $\dot{\mathbf{n}}$.

In frame $\left(\mathbf{q}, \mathbf{q}^{\perp}, \mathbf{n}\right)$, we can write any vector $\mathbf{v}$ as

$$
\mathbf{v}=(\mathbf{q} \cdot \mathbf{v}) \mathbf{q}+\left(\mathbf{q}^{\perp} \cdot \mathbf{v}\right) \mathbf{q}^{\perp}+(\mathbf{n} \cdot \mathbf{v}) \mathbf{n} .
$$

To find $\dot{\mathbf{n}}$, we apply Equation (11) with $\mathbf{v}=\dot{\mathbf{n}}$, and calculate the coefficients $(\mathbf{q} \cdot \dot{\mathbf{n}}),\left(\mathbf{q}^{\perp} \cdot \dot{\mathbf{n}}\right)$, and $(\mathbf{n} \cdot \dot{\mathbf{n}})$. In the inertial frame, define the rotation matrix of the rigid body as $\mathbf{g}=$ $\left[\mathbf{q}, \mathbf{q}^{\perp}, \mathbf{n}\right] \in S O(3)$. Define a skew symmetric matrix $S(\omega)$, in which $\omega \in R^{3}$ is the angular velocity of the rigid body. Then, we have $\dot{\mathbf{g}}=S(\omega) \mathbf{g}$, which suggests that $\dot{\mathbf{n}}=\omega \times \mathbf{n}$. Since the speed of $\mathbf{r}_{i}$ along directions $\mathbf{q}$ and $\mathbf{q}^{\perp}$ are zero for the rigid body, we conclude that $\omega$ is confined in plane $M$.

For the velocity of the agent in the inertial frame, $\mathbf{v}_{i}-\mathbf{v}_{c}$ satisfies

$$
\mathbf{v}_{i}-\mathbf{v}_{c}=\omega \times\left(\mathbf{r}_{i}-\mathbf{r}_{c}\right) .
$$

Then, we have

$$
\left(v_{i, \mathbf{n}}-v_{c, \mathbf{n}}\right) \mathbf{n}=\omega \times\left(\mathbf{r}_{i}-\mathbf{r}_{c}\right) .
$$

By taking inner product with $\mathbf{n}$ on both sides of Equation (13), we obtain

$$
v_{i, \mathbf{n}}-v_{c, \mathbf{n}}=\omega \times\left(\mathbf{r}_{i}-\mathbf{r}_{c}\right) \cdot \mathbf{n} .
$$

Define $\omega_{i}=\omega \times\left(\mathbf{r}_{i}-\mathbf{r}_{c}\right) \cdot \mathbf{n}$. We have

$$
\omega_{i}=-\left(\mathbf{r}_{i}-\mathbf{r}_{c}\right) \cdot(\omega \times \mathbf{n})=-\left(\mathbf{r}_{i}-\mathbf{r}_{c}\right) \cdot \dot{\mathbf{n}} .
$$

For simplicity, we constrain the three agents to form an equilateral triangle, which means that $\left\|\mathbf{r}_{1}-\mathbf{r}_{2}\right\|=\| \mathbf{r}_{2}-$ $\mathbf{r}_{3}\|=\| \mathbf{r}_{3}-\mathbf{r}_{1} \|$. In this case, $\mathbf{q}$ and $\mathbf{q}^{\perp}$ can be chosen as $\frac{\mathbf{r}_{2}-\mathbf{r}_{1}}{\left\|\mathbf{r}_{2}-\mathbf{r}_{1}\right\|}$ and $\frac{\mathbf{r}_{3}-\mathbf{r}_{c}}{\left\|\mathbf{r}_{3}-\mathbf{r}_{c}\right\|}$, respectively. We derive

$$
\omega_{3}=-\left\|\mathbf{r}_{3}-\mathbf{r}_{c}\right\| \frac{\mathbf{r}_{3}-\mathbf{r}_{c}}{\left\|\mathbf{r}_{3}-\mathbf{r}_{c}\right\|} \cdot \dot{\mathbf{n}}=-\left\|\mathbf{r}_{3}-\mathbf{r}_{c}\right\|\left(\mathbf{q}^{\perp} \cdot \dot{\mathbf{n}}\right),
$$

and

$\omega_{2}-\omega_{1}=-\left\|\mathbf{r}_{2}-\mathbf{r}_{1}\right\| \frac{\mathbf{r}_{2}-\mathbf{r}_{1}}{\left\|\mathbf{r}_{2}-\mathbf{r}_{1}\right\|} \cdot \dot{\mathbf{n}}=-\left\|\mathbf{r}_{2}-\mathbf{r}_{1}\right\|(\mathbf{q} \cdot \dot{\mathbf{n}})$,

which produces $\mathbf{q}^{\perp} \cdot \dot{\mathbf{n}}=-\frac{\omega_{3}}{\left\|\mathbf{r}_{3}-\mathbf{r}_{c}\right\|}$ and $\mathbf{q} \cdot \dot{\mathbf{n}}=-\frac{\omega_{2}-\omega_{1}}{\left\|\mathbf{r}_{2}-\mathbf{r}_{1}\right\|}$. In addition, since $\mathbf{n}$ is a unit vector, we have $\mathbf{n} \cdot \dot{\mathbf{n}}=0$. Thus, $\mathbf{n}$ has the form

$$
\dot{\mathbf{n}}=-\frac{\omega_{2}-\omega_{1}}{\left\|\mathbf{r}_{2}-\mathbf{r}_{1}\right\|} \mathbf{q}-\frac{\omega_{3}}{\left\|\mathbf{r}_{3}-\mathbf{r}_{c}\right\|} \mathbf{q}^{\perp} .
$$

From Equation (14), we have $\omega_{i}=v_{i, \mathbf{n}}-v_{c, \mathbf{n}}$. Since the field $f(\mathbf{r})$ is at least class $C^{1}$, then, from the Taylor expansion, we have

$$
v_{i, \mathbf{n}}=k\left(f\left(\mathbf{r}_{c}\right)+\nabla f\left(\mathbf{r}_{c}\right) \cdot\left(\mathbf{r}_{i}-\mathbf{r}_{c}\right)\right)+C+\text { H.O.T, }
$$

where H.O.T represents higher order terms. In addition,

$$
\begin{aligned}
& v_{c, \mathbf{n}}=\frac{k}{3} \sum_{i=1}^{3} v_{i, \mathbf{n}}=\frac{1}{3} \sum_{i=1}^{3} f\left(\mathbf{r}_{i}\right)+C \\
& =k f\left(\mathbf{r}_{c}\right)+\frac{k}{3} \nabla f\left(\mathbf{r}_{c}\right) \cdot\left(\sum_{i=1}^{3} \mathbf{r}_{i}-3 \mathbf{r}_{c}\right)+C=k f\left(\mathbf{r}_{c}\right)+C .
\end{aligned}
$$

Therefore, if the higher order terms are insignificant compared to the first and second order term in Equation (19), we derive

$$
\begin{aligned}
\omega_{i} & =v_{i, \mathbf{n}}-v_{c, \mathbf{n}}=k \nabla f\left(\mathbf{r}_{c}\right) \cdot\left(\mathbf{r}_{i}-\mathbf{r}_{c}\right) \\
& =k\left\|\nabla f\left(\mathbf{r}_{c}\right)\right\|\left(\frac{\nabla f\left(\mathbf{r}_{c}\right)}{\left\|\nabla f\left(\mathbf{r}_{c}\right)\right\|} \cdot\left(\mathbf{r}_{i}-\mathbf{r}_{c}\right)\right) \\
& =k\left\|\nabla f\left(\mathbf{r}_{c}\right)\right\|\left(\mathbf{N} \cdot\left(\mathbf{r}_{i}-\mathbf{r}_{c}\right)\right),
\end{aligned}
$$

which leads to

$$
\begin{aligned}
\omega_{2}-\omega_{1} & =k\left\|\nabla f\left(\mathbf{r}_{c}\right)\right\|\left(\mathbf{N} \cdot\left(\mathbf{r}_{2}-\mathbf{r}_{1}\right)\right) \\
& =k\left\|\nabla f\left(\mathbf{r}_{c}\right)\right\|\left\|\mathbf{r}_{2}-\mathbf{r}_{1}\right\|\left(\mathbf{N} \cdot \frac{\mathbf{r}_{2}-\mathbf{r}_{1}}{\left\|\mathbf{r}_{2}-\mathbf{r}_{1}\right\|}\right) \\
& =k\left\|\nabla f\left(\mathbf{r}_{c}\right)\right\|\left\|\mathbf{r}_{2}-\mathbf{r}_{1}\right\|(\mathbf{N} \cdot \mathbf{q}),
\end{aligned}
$$


and

$$
\begin{aligned}
\omega_{3} & =k\left\|\nabla f\left(\mathbf{r}_{c}\right)\right\|\left\|\mathbf{r}_{3}-\mathbf{r}_{c}\right\|\left(\mathbf{N} \cdot \frac{\mathbf{r}_{3}-\mathbf{r}_{c}}{\left\|\mathbf{r}_{3}-\mathbf{r}_{c}\right\|}\right) \\
& =k\left\|\nabla f\left(\mathbf{r}_{c}\right)\right\|\left\|\mathbf{r}_{3}-\mathbf{r}_{c}\right\|\left(\mathbf{N} \cdot \mathbf{q}^{\perp}\right) .
\end{aligned}
$$

Substitute Equations (22) and (23) into Equation (18), we obtain $\dot{\mathbf{n}}=-k\left\|\nabla f\left(\mathbf{r}_{c}\right)\right\|\left((\mathbf{N} \cdot \mathbf{q}) \mathbf{q}+\left(\mathbf{N} \cdot \mathbf{q}^{\perp}\right) \mathbf{q}^{\perp}\right)$. Therefore, using (10), the closed-loop system is derived as

$$
\frac{d(\mathbf{N} \cdot \mathbf{n})}{d t}=-k\left\|\nabla f\left(\mathbf{r}_{c}\right)\right\|\left(1-(\mathbf{N} \cdot \mathbf{n})^{2}\right)+\dot{\mathbf{N}} \cdot \mathbf{n},
$$

in which $\nabla f\left(\mathbf{r}_{c}\right)$ is time-varying as $\mathbf{r}_{c}$ changes.

For simplicity, denote $\theta=\mathbf{N} \cdot \mathbf{n}$ and $\delta=\dot{\mathbf{N}} \cdot \mathbf{n}$. Since $\mathbf{N}$ and $\mathbf{n}$ are unit vectors, we have $-1 \leq \theta \leq 1$. System (24) can be rewritten as

$$
\dot{\theta}=-k\left\|\nabla f\left(\mathbf{r}_{c}\right)\right\|\left(1-\theta^{2}\right)+\delta .
$$

Let $h(t, \theta, \delta) \triangleq-k\left\|\nabla f\left(\mathbf{r}_{c}\right)\right\|\left(1-\theta^{2}\right)+\delta$. If $\left\|\nabla f\left(\mathbf{r}_{c}\right)\right\| \neq$ 0 , the unforced system $h(t, \theta, 0)$ has two equilibriums $\theta=1$ and $\theta=-1$. We will prove that if we set $\theta(t)<0$ at $t=0$, $\theta(t)$ will always be less than zero as $t \rightarrow \infty$. We have the following proposition.

Proposition 3.1: Consider the time-varying system (25). Assume $\|\nabla f(\mathbf{r})\|$ is bounded below along the trajectory of the formation center. That is, $\|\nabla f(\mathbf{r})\| \geq \varepsilon_{c}$, in which $\varepsilon_{c}>0$ is a constant. If at $t=0$, we set $\theta(0)=\mathbf{N} \cdot \mathbf{n}<0$, then, as $t \rightarrow$ $\infty$, the equilibrium $\theta=-1$ of the unforced system $h(t, \theta, 0)$ is asymptotically stable for all initial conditions satisfying $-1 \leq \theta(0)<0$. Furthermore, for all initial conditions $-1 \leq$ $\theta(0)<0$ and $|\delta|<k \varepsilon \varepsilon_{c}$, in which $0<\varepsilon<1$ is a constant, the system (25) is input-to-state stable on the interval $\theta \in$ $[-1,0)$.

Proof: Define a new variable $\beta=\theta+1$, then, $0 \leq \beta \leq$ 2. System (25) becomes

$$
\dot{\beta}=k\left\|\nabla f\left(\mathbf{r}_{c}\right)\right\|\left(\beta^{2}-2 \beta\right)+\delta .
$$

$\beta=0$ and $\beta=2$ are two equilibriums of the unforced system $h(t, \beta, 0)$, which correspond to $\mathbf{N} \cdot \mathbf{n}=-1$ and 1 , respectively. Since $\mathbf{N}$ is perpendicular to $\mathbf{N}$, we have $\dot{\mathbf{N}} \cdot \mathbf{n}=0$ when $\mathbf{N} \cdot \mathbf{n}=-1$ or 1 . Therefore, $\delta=0$ when $\beta=0$ or $\beta=2$, which implies that $\beta$ remains in $[0,2]$ even though there is perturbation $\delta$.

To prove the input-to-state stability of the equilibrium $\beta=$ 0 , we define a Lyapunov candidate function on the interval $0 \leq \beta \leq 2$ as

$$
V(\beta)=\frac{1}{2} \beta^{2} .
$$

We have $V(0)=0$, and $V(\beta)>0$ for $0<\beta \leq 2$. We derive

$$
\dot{V}(\beta)=\dot{\beta} \beta=-k\left\|\nabla f\left(\mathbf{r}_{c}\right)\right\|(2-\beta) \beta^{2}+\beta \delta .
$$

When $\beta=1$, from the assumptions $\|\nabla f(\mathbf{r})\| \geq \varepsilon_{c}$ and $|\delta|<$ $k \varepsilon \varepsilon_{c}$, Equation (28) becomes

$$
\dot{V}(1)=-k\left\|\nabla f\left(\mathbf{r}_{c}\right)\right\|+\delta<-k \varepsilon_{c}+k \varepsilon \varepsilon_{c}<0 .
$$

Therefore, if at $t=0$, we set $0 \leq \beta(0)<1$, then, since $V(\beta(t))$ is a monotonic function of $\beta$ on the interval $0 \leq \beta<$ 1 and bounded below by zero, the trajectory of the system will stay in a compact sub-level set of the Lyapunov function, implying $0 \leq \beta(t)<1$ for all $t>0$. Thus, we are interested in the case when $0 \leq \beta<1$. If $0 \leq \beta<1$ for all $t$, then, $\beta-2 \leq-\beta$. We derive

$$
\begin{aligned}
\dot{V}(\beta) & =-k\left\|\nabla f\left(\mathbf{r}_{c}\right)\right\|(2-\beta) \beta^{2}+\beta \delta \\
& \leq-k\left\|\nabla f\left(\mathbf{r}_{c}\right)\right\| \beta^{3}+\beta \delta \\
& =-(1-\varepsilon) k\left\|\nabla f\left(\mathbf{r}_{c}\right)\right\| \beta^{3}-\varepsilon k\left\|\nabla f\left(\mathbf{r}_{c}\right)\right\| \beta^{3}+\beta \delta \\
& \leq-(1-\varepsilon) k\left\|\nabla f\left(\mathbf{r}_{c}\right)\right\| \beta^{3}, \quad \forall\|\beta\| \geq \sqrt{\frac{|\delta|}{k \varepsilon \varepsilon_{c}}}
\end{aligned}
$$

The assumption $|\delta|<k \varepsilon \varepsilon_{c}$ implies that $\sqrt{\frac{|\delta|}{k \varepsilon \varepsilon_{c}}}<1$, which guarantees the set $\beta \in\left[\sqrt{\frac{|\delta|}{k \varepsilon \varepsilon_{c}}}, 1\right)$ is not empty.

Denote $\alpha_{1}(|\beta|)=\alpha_{2}(|\beta|)=\frac{1}{2}|\beta|^{2}$, which are class $K$ functions when $0 \leq|\beta|<1$. We have $\alpha_{1}(|\beta|) \leq V(\beta) \leq$ $\alpha_{2}(|\beta|)$. In addition, $W_{3}(\beta)=(1-\varepsilon) k\left\|\nabla f\left(\mathbf{r}_{c}\right)\right\| \beta^{3}$ is a continuous positive definite function on $0 \leq \beta<1$, and $\rho(|\delta|)=\sqrt{\frac{|\delta|}{k \varepsilon \varepsilon_{c}}}$ is a class $K$ function. Therefore, we have $\dot{V}(\beta) \leq-W_{3}(\beta), \forall \rho(|\delta|) \leq|\beta|<1$. Thus, according to Theorem 4.19 in [29], the system (25) is input-to-state stable over the interval $0 \leq \beta<1$. The input-to-state stability implies that the equilibrium $\beta=0$ of the unforced system $h(t, \beta, 0)$ is asymptotically stable with initial conditions starting from all points in $[0,1)$. In other words, $\theta(t)$ converges to -1 asymptotically as $t>\infty$.

Proposition 3.1 indicates that, without perturbation $\delta$, as $t \rightarrow \infty$, the moving direction of the rigid body will converge to be aligned with the opposite direction of the gradient of the field, thus, the rigid body moves towards a local minimum of the field. If the perturbation $\delta$ is bounded, the system is input-to-state stable. Hence, the moving direction of the rigid body will stay close to the opposite direction of the gradient if the perturbation $\delta$ is small.

\section{Generalization to N-agent Groups}

In [5], the design for controlling $\mathrm{N}$-agent groups was generalized from the two-agent case in $2 \mathrm{D}$. In $3 \mathrm{D}$, we can also generalize the results to $N$-agent groups. Instead of choosing a reference vector as in 2D case, we choose three agents from the $N$-agent group that determine a reference plane $M$ and define a right-handed coordinate frame $\left[\mathbf{q}, \mathbf{q}^{\perp}, \mathbf{n}\right]$ in the same way as in the three-agent case. Then, we decompose the velocity of each agent along directions of $\mathbf{q}, \mathbf{q}^{\perp}$, and $\mathbf{n}$. Equation (4) holds for the all the agents in the $\mathrm{N}$-agent group.

Unlike the three-agent group that remains a constant formation, the $N$-agent group is only controlled to maintain constant distances among agents along directions $\mathbf{q}$ and $\mathbf{q}^{\perp}$. As illustrated in Fig. 5, we denote the three agents that determines plane $M$ as $\mathbf{r}_{1}, \mathbf{r}_{2}$, and $\mathbf{r}_{3}$, and project all the other agents onto plane $M$. Notice that agents $\mathbf{r}_{1}, \mathbf{r}_{2}$, and $\mathbf{r}_{3}$ will be controlled to form an equilateral triangle. The black dots illustrate the positions of each agent, and the grey dots show the projections of agent $m, n, k$, and $l$ onto plane $M$. The 


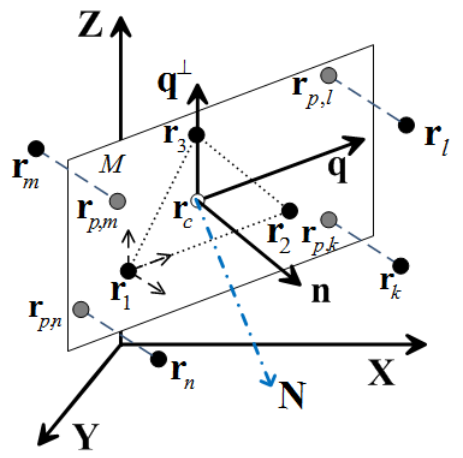

Fig. 5. Geometry of a $N$-agent group. Projections of the agents $\mathbf{r}_{k}, \mathbf{r}_{l}, \mathbf{r}_{m}$, and $\mathbf{r}_{n}$ onto the plane determined by $\mathbf{r}_{1}, \mathbf{r}_{2}$, and $\mathbf{r}_{3}$ are $\mathbf{r}_{p, k}, \mathbf{r}_{p, l}, \mathbf{r}_{p, m}$, and $\mathbf{r}_{p, n}$.

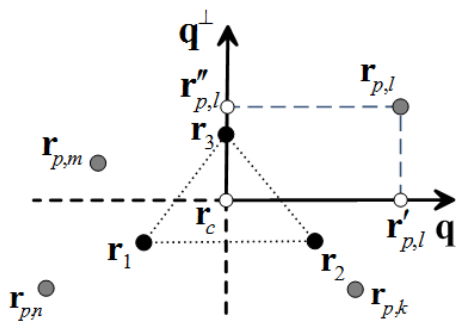

Fig. 6. Projections of $\mathbf{r}_{p, l}$ onto axis $\mathbf{q}$ and $\mathbf{q}^{\perp}$ are $\mathbf{r}_{p, l}^{\prime}$ and $\mathbf{r}_{p, l}^{\prime \prime}$.

projected positions are denoted by $\mathbf{r}_{p, i}, i=1, \cdots, N$. Notice that $\mathbf{r}_{p, 1}=\mathbf{r}_{1}, \mathbf{r}_{p, 2}=\mathbf{r}_{2}$, and $\mathbf{r}_{p, 3}=\mathbf{r}_{3}$.

Similar to the three-agent case, we project $\mathbf{r}_{p, i}, i=1, \cdots, N$ onto $\mathbf{q}$ and $\mathbf{q}^{\perp}$, and denote the projected positions as $\mathbf{r}_{p, i}^{\prime}$ and $\mathbf{r}_{p, i}^{\prime \prime}$, respectively. In Fig. 6, we demonstrate the projections of agent $\mathbf{r}_{p, l}$ onto $\mathbf{q}$ and $\mathbf{q}^{\perp}$. For agent $i$, we also define sets $N_{i}$ and $N_{i}^{\perp}$ that contain the indices of the neighboring agents along directions $\mathbf{q}$ and $\mathbf{q}^{\perp}$. For example, as shown in Fig. $6, N_{1}=\{m, 3\}, N_{k}=\{2, l\}, N_{3}^{\perp}=\{m, l\}$, and $N_{2}^{\perp}=\{k, 1, l\}$. Then, we design

$$
\begin{aligned}
v_{i, \mathbf{q}} & =k_{1} \sum_{j \in N_{i}}\left(\left(\mathbf{r}_{p, j}-\mathbf{r}_{p, i}\right) \cdot \mathbf{q}-a_{j, i}^{0}\right), \\
v_{i, \mathbf{q}^{\perp}} & =k_{2} \sum_{j \in N_{i}^{\perp}}\left(\left(\mathbf{r}_{p, j}-\mathbf{r}_{p, i}\right) \cdot \mathbf{q}^{\perp}-b_{j, i}^{0}\right),
\end{aligned}
$$

where $k_{1}>0$ and $k_{2}>0$ are constants, $a_{i, j}^{0}=-a_{j, i}^{0}$, and $b_{i, j}^{0}=$ $-b_{j, i}^{0}$ are desired distances between $\mathbf{r}_{p, i}^{\prime}$ and $\mathbf{r}_{p, j}^{\prime}$, and $\mathbf{r}_{p, i}^{\prime \prime}$ and $\mathbf{r}_{p, j}^{\prime \prime}$, respectively. Using similar methods as in the threeagent case, we can prove that the relative distance from $\mathbf{r}_{p, i}^{\prime}$ to $\mathbf{r}_{p, j}^{\prime}, i \neq j$, converges to a constant $a_{i j}^{0}$, and the relative distance from $\mathbf{r}_{p, i}^{\prime \prime}$ to $\mathbf{r}_{p, j}^{\prime \prime}, i \neq j$, converges to a constant $b_{i j}^{0}$.

Along direction n, we still use Equation (5) as the control for each sensing agent. Notice that, under this design, the distance between two agents $i$ and $j$ along direction $\mathbf{n}$ is determined by $f\left(\mathbf{r}_{i}\right)-f\left(\mathbf{r}_{j}\right)$, which may not be a constant. Therefore, the $N$-agent group can not be controlled as a rigid body in 3D. However, as we discussed, the projections of the agents onto plane $M$ can be controlled to maintain a constant formation, which can be considered as a rigid body.
Therefore, the convergence proof in the three-agent case can still be applied. Following similar arguments, we can prove that the moving direction of the plane determined by agents $\mathbf{r}_{1}, \mathbf{r}_{2}$, and $\mathbf{r}_{3}$ will be aligned with the opposite direction of the gradient of field $f(\mathbf{r})$.

\section{Simulation Results in Smooth fields}

To demonstrate our source-seeking strategy, we simulate three sensing agents moving in a smooth field $f(\mathbf{r})=\mathbf{r}^{T} \mathbf{r}$ under the control laws (5), (6), and (7), in which we choose $k=1, C=0$, and $k_{1}=k_{2}=1$. The field has a minimum located at $\mathbf{r}=(0,0,0)$. The initial positions of the agents are $\mathbf{r}_{1}=[0.9,0.8,0.8], \mathbf{r}_{2}=[0.8,0.9,0.8]$, and $\mathbf{r}_{3}=[0.85,0.85,0.85]$. The desired distances among agents are $a_{31}^{0}=-a_{23}^{0}=0.05$ and $b_{31}^{0}=b_{32}^{0}=0.1 \frac{\sqrt{3}}{2}$. As illustrated in Fig. 7, the three agents (red, green, and blue dots) converge to a constant formation, and move towards the minimum of the field. The moving direction of the center of the formation converges to the opposite direction of gradient directions. The black dots indicates the trajectory of the formation center.

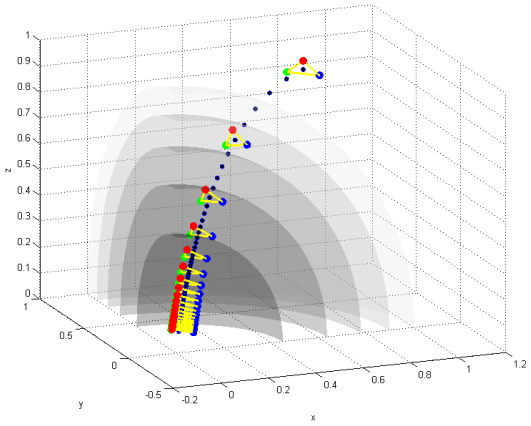

Fig. 7. Simulation results of three agents locating a local minimum of a smooth field.

\section{Plume Tracking in Turbulent Flow}

In the control (5), the field value $f(\mathbf{r})$ needs to be estimated at every step by the agents based on the measurements of the turbulent field. In this section, we present an approach that estimates the rates $\lambda_{12}$ and $\lambda_{21}$ so that the smooth field $f(\mathbf{r})$ can be generated along the trajectories of each agent, then, we apply the source-seeking strategy in the smooth field $f(\mathbf{r})$ to control the agents in a turbulent field to track a plume source.

\section{A. Plume Tracking Algorithm}

Along its trajectory, a sensing agent takes measurements of a turbulent field, then compares the measurements with a given threshold to determine if there is a spike. A measurement being greater than the threshold indicates that a spike is detected. Suppose the current time is $t$ and the $j$-th spike has been detected along the trajectory of the $i$ th agent. Denote $T_{j, i}$ and $T_{j, i}^{\prime}$ as the beginning and ending time of the $j$-th 
spike, respectively. Then, the rate of state transitions can be estimated by

$$
\hat{\lambda}_{12, i}(t)=\frac{1}{T_{j, i}-T_{j-1, i}^{\prime}}, \quad \hat{\lambda}_{21, i}(t)=\frac{1}{T_{j, i}^{\prime}-T_{j, i}} .
$$

Considering the noise or imperfect design of the sensor, $\hat{\lambda}_{12, i}(t)$ and $\hat{\lambda}_{21, i}(t)$ in Equation (33) represent noisy estimates of the rates. Using the estimates $\hat{\lambda}_{12, i}(t)$ and $\hat{\lambda}_{21, i}(t)$, the mobile agent can compute a noisy estimate of $f\left(\mathbf{r}_{i}\right)$ as

$$
\hat{f}\left(\mathbf{r}_{i}\right)=\frac{\hat{\lambda}_{12, i}+\hat{\lambda}_{21, i}}{\hat{\lambda}_{12, i}}=f\left(\mathbf{r}_{i}\right)+w\left(\mathbf{r}_{i}\right),
$$

in which $w\left(\mathbf{r}_{i}\right)$ represents the error term that may be induced by estimation error or measurement noise. Therefore, Equation (5) becomes

$$
v_{i, \mathbf{n}}=k \hat{f}\left(\mathbf{r}_{i}\right)+C .
$$

The derivation and convergence proof in Section III assumes no noise in field value $f\left(\mathbf{r}_{i}\right)$. However, with the presence of the noise $w\left(\mathbf{r}_{i}\right)$, uncertainties in the moving direction arise. We have analyzed the situation when noise is present in $2 \mathrm{D}$ case [5]. The extension to 3D case will be analyzed in our future work.

Consider the implementation in practise. We discretize the system and use the designed velocities (6), (7), and (35) as path planning algorithm for the agent group. Let us denote by $\mathbf{r}_{i, k}=\mathbf{r}_{i}\left(t_{k}\right)$ the position of the $i$ th agent at time $t_{k}$ and by $h$ the time step size. Then, the agents will seek the plume source based on the following path-planning algorithm:

Algorithm 4.1: Repeat the following steps for $i=1,2,3$. At location $\mathbf{r}_{i, k}$, the $i$ th sensing agent

1) takes measurements of the turbulent field values at each time step for a finite time $T$ called the "waiting time".

2) estimates $\hat{\lambda}_{12, i}$ and $\hat{\lambda}_{21, i}$ based on the measurements, estimates $\hat{f}\left(\mathbf{r}_{i}\right)$ based on Equation (34), and determines the velocity $\mathbf{v}_{i, k}$ based on Equations (6), (7), and (35), which generates a planned trajectory.

3) moves forward along the trajectory over a finite motion horizon time $\tau$ so that $\mathbf{r}_{i, k+1}=\mathbf{r}_{i, k}+\frac{\tau}{h} \mathbf{v}_{i, k}$.

4) stops if either the mean of $\hat{f}\left(\mathbf{r}_{i}\right)$ estimated by the agents is sufficiently close to 1 , indicating the vicinity of the source, or the distance of the formation center from the source is $\frac{1}{N} \sum_{i=1}^{N}\left\|\mathbf{r}_{i}-\mathbf{r}_{0}\right\| \leq \varepsilon$, where $\varepsilon=5 \mathrm{~cm}$ in the simulation; otherwise, repeat from step 1 .

\section{B. Simulation Results in Turbulent Flow}

We now simulate the plume tracking algorithm 4.1 in a 3D simulated turbulent flow field using three sensing agents. Choose $k_{\lambda}=f_{r} d_{r}$ and $k_{\lambda}^{\prime}=\frac{f_{r}}{d_{r}}$, in which the reference frequency $f_{r}=4 \mathrm{~Hz}$ and reference distance $d_{r}=150 \mathrm{~cm}$. These parameters determine the jump rates $\lambda_{12}, \lambda_{21}$ for spikes in the simulation, which agree with our previous work [23]. Then, at each location in the plume field, a time series of spikes is generated using Equation (1). Each sensing agent measures this series of spikes at its current position for the waiting time $T$ as described in Algorithm 4.1, then it estimates the field $f(\mathbf{r})$.

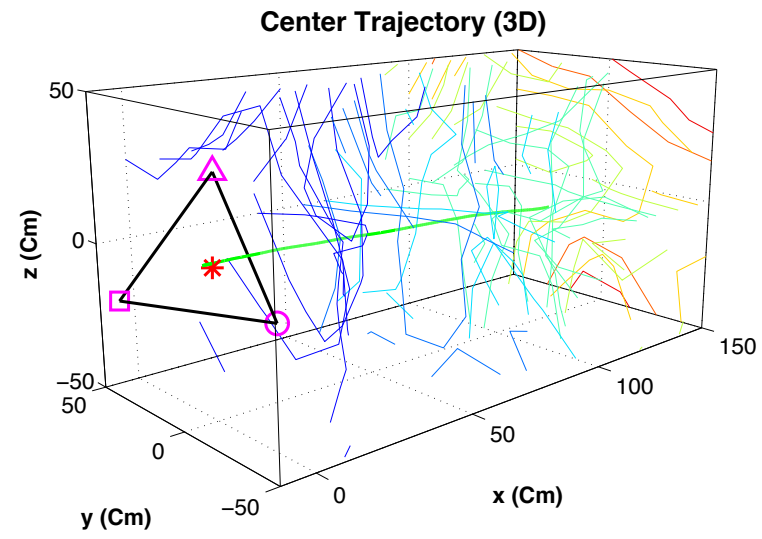

Fig. 8. The trajectory of the center of three mobile sensing agents in a plume tracking simulation in 3D. The plume source is located at $(0,0,0)$ and the agents are deployed at $(150,55,-30),(150,5,-30)$, and $(150,30,-30+25 \sqrt{3})$. The waiting time $T=50 \mathrm{~s}$ at each position and the agents move forward for $\tau=1 s$ after the velocities are determined.

According to Algorithm 4.1, we simulate three mobile sensing agents in the simulated turbulent fluid field using control laws (6), (7), and (35). For the controller parameters, we set $k=3, C=-3$, and $k_{1}=k_{2}=1$. The initial positions of the simulated agents are $\mathbf{r}_{1}^{0}=\left(150,30+a_{13}^{0},-30\right), \mathbf{r}_{2}^{0}=$ $\left(150,30+a_{23}^{0},-30\right)$, and $\mathbf{r}_{3}^{0}=\left(150,30,-30+b_{13}^{0}\right)$, where $a_{13}^{0}=-a_{23}^{0}=25 \mathrm{~cm}$ and $b_{13}^{0}=b_{23}^{0}=25 \sqrt{3} \mathrm{~cm}$. The agents estimate the averaged plume field for the waiting time $T$ at their current position, then move forward for $\tau=1 \mathrm{~s}$. Each agent neither needs the entire field information nor shares their information with other agents to navigate. Instead, they use the spike parameters $\hat{\lambda}_{12}, \hat{\lambda}_{21}$ only at their current positions to compute $\hat{f}\left(\mathbf{r}_{i}\right)$ and $\mathbf{v}_{i}$ in the algorithm, which takes very small computational cost and hence enables realtime implementation.

Simulation results are illustrated in Figs. 8 and 9. After estimating $\hat{\lambda}_{12, i}$ and $\hat{\lambda}_{21, i}$ using Equation (33), each agent computes the velocity and moves forward. Fig. 8 shows the trajectory (the green line) of the center of the three agents towards the source. The ending positions of each of the three agents are marked by purple square, circle, and triangle markers, respectively. The relative displacements between the agents are plotted as the black lines connecting the ending positions of the three agents. We see that the relative distances between the agents are maintained till the end. In Fig. 9, we can observe that the center of the agents smoothly converges to the source on both $\mathrm{x}-\mathrm{y}$ and $\mathrm{x}-\mathrm{z}$ planes.

\section{CONCLUSIONS}

In this paper, we develop a distributed source-seeking algorithm using mobile sensor networks in 3D fields without explicit gradient estimation. The velocity of each agent is designed using only the measurements taken by the agent and the relative positions to the neighboring agents. We prove that, without perturbation, the moving direction of the agent group will converge to the opposite direction of the field 

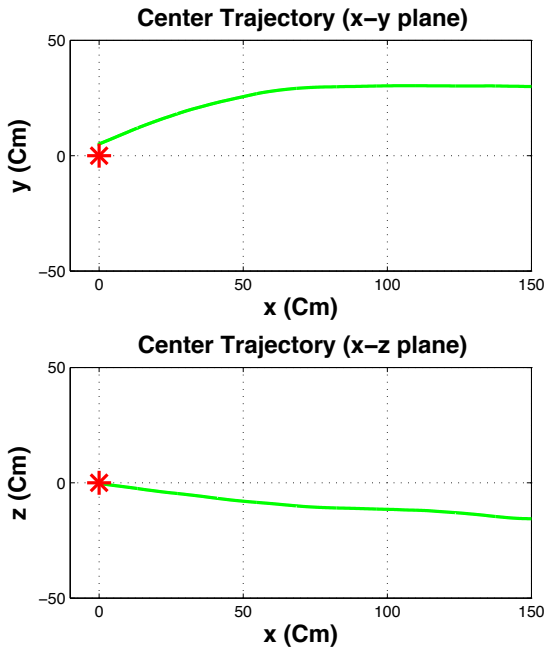

Fig. 9. Top-view (x-y plane) and side-view ( $x-z$ plane) trajectory images of the plume tracking simulation in Fig. 8. The center of three mobile sensing agents is smoothly converging to the plume source using Algorithm 4.1 with $T=50 \mathrm{~s}$ and $\tau=1 \mathrm{~s}$.

gradient, and the system is input-to-state stable when the perturbation is small. The strategy is applied to mobile sensor networks in turbulent fields tracking a plume source. The application of the algorithm is enabled by the transformation of a turbulent field detected by sensing agents to a smoother field sharing the same source as the turbulent field. Future work includes analysing the control strategy with the presence of noise in the measurements, and verifying the plume tracking algorithm using real experimental data.

\section{REFERENCES}

[1] P. Ogren, E. Fiorelli, and N. E. Leonard, "Cooperative control of mobile sensor networks: Adaptive gradient climbing in a distributed environment," IEEE Transactions on Automatic Control, vol. 49, no. 8, pp. 1292-1302, 2004.

[2] J. Cortes, "Distributed gradient ascent of random fields by robotic sensor networks," in Proc. 46th IEEE Conf. on Decision and Control, 2007, pp. 3120-3126.

[3] B. J. Moore and C. C. de Wit, "Source seeking via collaborative measurements by a circular formation of agents," in Proc. of 2010 American Control Conference, 2010, pp. 6417-6422.

[4] W. Wu and F. Zhang, "Robust cooperative exploration with a switching strategy," IEEE Transactions on Robotics, vol. 28, no. 4, pp. 828-839, 2012.

[5] W. Wu, I. D. Couzin, and F. Zhang, "Bio-inspired source seeking with no explicit gradient estimation," in Proc. of IFAC Workshop on Distributed Estimation and Control in Networked System, 2012, pp. 240-245.

[6] E. Burian, D. Yoerger, A. Bradley, and H. Singh, "Gradient search with autonomous underwater vehicles using scalar measurements," in Proceedings of the 1996 Symposium on Autonomous Underwater Vehicle Technology, 1996, pp. 86 - 98.

[7] N. Ghods and M. Krstic, "Speed regulation in steering-based source seeking," Automatica, vol. 46, pp. 452-459, 2010.

[8] S. J. Liu and M. Krstic, "Stochastic source seeking for nonholonomic unicycle," Automatica, vol. 46, pp. 1443-1453, 2010.

[9] A. Matveev, H. Teimoori, and A. V. Savkin, "Navigation of a unicyclelike mobile robot for environmental extremum seeking," Automatica, vol. 47, no. 1, pp. 85-91, 2011.

[10] S. Azuma, M. Sakar, and G. Pappas, "Stochastic source seeking by mobile robots," IEEE Transactions on Automatic Control, vol. 57, no. 9, pp. 2308-2321, 2012.
[11] J. Cochran, A. Siranosian, N. Ghods, and M. Krstic, "3-d source seeking for underactuated vehicles without position measurement," IEEE Transactions on Robotics, vol. 25, no. 1, pp. 117-129, 2009.

[12] A. Berdahl, C. Torney, C. Ioannou, J. Faria, and I. Couzin, "Emergent sensing of complex environments by mobile animal groups," Science, vol. 339, no. 6119, pp. 574-576, 2013.

[13] D. R. Webster and M. J. Weissburg, "Chemosensory guidance cues in a turbulent chemical odor plume," Limnology and Oceanography, vol. 46, no. 5, pp. 1034-1047, 2001

[14] _ - "The hydrodynamics of chemical cues among aquatic organisms," Annual Review of Fluid Mechanics, vol. 41, no. 1, pp. 73-90, 2009.

[15] G. Kowadlo and R. a. Russell, "Robot odor localization: A taxonomy and survey," The International Journal of Robotics Research, vol. 27, no. 8, pp. 869-894, 2008.

[16] C. Lytridis, G. Virk, Y. Rebour, and E. Kadar, "Odor-based navigational strategies for mobile agents," Adaptive Behavior, vol. 9, no. 3-4, pp. 171-187, 2001.

[17] R. Russella, A. Bab-Hadiashar, R. L. Shepherd, and G. G. Wallace, "Acomparison of reactive robot chemotaxisalgorithms," Robotics and Autonomous Systems, vol. 45, no. 2, pp. 83-97, 2003.

[18] M. J. Weissburg and D. B. Dusenbery, "Behavioral observations and computer simulations of blue crab movement to a chemical source in a controlled turbulent flow," Experimental Biology, vol. 205, pp. 3387-3398, 2002.

[19] D. R. Webster, K. Y. Volyanskyy, and M. J. Weissburg, "Bioinspired algorithm for autonomous sensor-driven guidance in turbulent chemical plumes," Bioinspiration \& Biomimetics, vol. 7, no. 3, p. 036023, 2012.

[20] J. A. Farrell, P. Shuo, and W. Li, "Chemical plume tracing via an autonomous underwater vehicle," IEEE Journal of Oceanic Engineering, vol. 30, no. 2, pp. 428-442, 2005.

[21] W. Li, J. A. Farrell, S. Pang, and R. M. Arrieta, "Moth-inspired chemical plume tracing on an autonomous underwater vehicle," IEEE Transactions on Robotics, vol. 22, no. 2, pp. 292-307, 2006.

[22] P. Pyk, S. B. i. Badia, U. Bernardet, P. Knsel, M. Carlsson, J. Gu, E. Chanie, B. S. Hansson, T. C. Pearce, and P. F. M. J. Verschure, "An artificial moth: Chemical source localization using a robot based neuronal model of moth optomotor anemotactic search," Autonomous Robots, vol. 20, no. 3, pp. 197-213, 2006.

[23] D. Chang, W. Wu, D. Webster, M. Weissburg, and F. Zhang, "A bio-inspired plume tracking algorithm for mobile sensing swarms in turbulent flow," in In Proc. IEEE International Conference on Robotics and Automation, 2013.

[24] E. W. Justh and P. S. Krishnaprasad, "Natural frames and interacting particles in three dimensions," in Proc. of 44th IEEE Conf. on Decision and Control and the European Control Conference. IEEE, 2005, pp. 2841-2846.

[25] F. Zhang and N. E. Leonard, "Cooperative control and filtering for cooperative exploration," IEEE Transactions on Automatic Control, vol. 55 , no. 3, pp. 650-663, 2010.

[26] F. Zhang, "Geometric cooperative control of particle formations," IEEE Transactions on Automatic Control, vol. 55, no. 3, pp. 800-803, 2010.

[27] D. R. Webster, K. Y. Volyanskyy, and M. J. Weissburg, "Sensorymediated tracking behavior in turbulent chemical plumes," in 7th International Symposium on Turbulence and Shear Flow Phenomena, Ottawa, Canada, 2011.

[28] W. Wu and F. Zhang, "Cooperative exploration of level surfaces of three dimentional scalar fields," Automatica, vol. 47(9), pp. 20442051, 2011.

[29] H. Khalil, Nonlinear Systems, 3rd Ed. New Jersey: Prentice Hall, 2001. 